\title{
Critical Sensitivity in Driven Nonlinear Threshold Systems
}

\author{
Xiaohui Zhang ${ }^{1}$, Xianghong Xu ${ }^{1}$, Haiyin Wang ${ }^{1}$, Mengfen XiA ${ }^{1,2}$, \\ FUJIU KE ${ }^{1,3}$, and YILONG BAI ${ }^{1}$
}

\begin{abstract}
Rupture in heterogeneous brittle media, including earthquakes, can be regarded as complicated phenomena in driven nonlinear threshold systems. It displays catastrophe transition and sample-specificity, which results in difficulty of rupture prediction. Our numerical simulations indicate that critical sensitivity might be a common precursor of catastrophe transition and thus give a clue to catastrophe prediction. In this paper we present an analytical examination of critical sensitivity in driven nonlinear threshold systems, based on mean field approximation and damage relaxation time model. The result suggests that critical sensitivity is in reality a common feature prior to catastrophe transition in driven nonlinear threshold systems, with disordered mesoscopic heterogeneity. This result seems to be supported by rock experiments.
\end{abstract} system.

Key words: Critical sensitivity, catastrophe transition, sample-specificity, driven nonlinear threshold

\section{Introduction}

Damage and rupture in disordered heterogeneous brittle media, such as rocks and the earth's crust, present complexity (MEAKIN, 1991; CurRan, 1997; CurTin, 1997; Sahimi and Ababi, 1993; Ben-Zion and Sammis 2003; Jaume, and Sykes 1999; RUNDle et al., 2000; TiAmpo et al., 2000; BAI et al., 1994; XiA et al., 1996, 1997, 2000), especially it displays catastrophe transition and sample-specificity. Firstly, the rupture appears as a transition from globally stable accumulation of mesoscopic damage to catastrophic rupture (BAI et al., 1994; XIA et al., 2000; WeI et al., 2000). Secondly, the behavior of catastrophe transition in macroscopically identical systems exhibits macroscopic uncertainty or sample-specificity (XIA et al., 1996, 1997, 2000; WEI et al., 2000). Consequently, it is insufficient to represent catastrophe of a system

${ }^{1}$ State Key Laboratory of Nonlinear Mechanics, Institute of Mechanics, Chinese Academy Of Sciences, Beijing 100080, China.

E-mail: Zhangxh@1nm.imech.ac.cn;xxh@1nm.imech.ac.cn;why@1nm.imech.ac.cn;baiyl@1nm.imech.ac.cn

${ }^{2}$ Department of Physics, Peking University, Beijing 100871, China.

E-mail: xiam@lnm.imech.ac.cn

${ }^{3}$ Department of Applied Physics, Beijing University of Aeronautics and Astronautics, Beijing 100083, China. E-mail: kefj@lnm.imech.ac.cn 
by merely its macroscopically average properties. The behavior of catastrophe may sensitively depend on the details of disordered mesoscopic heterogeneity which is usually difficult, even impossible, to be dealt with. Such complexity results in difficulty of rupture prediction (WEI et al., 2000, WYSS et al., 1997; GARCIMARTIN et al., 1997).

To search the universal features of catastrophe transition is of genuine importance to rupture prediction. Our numerical simulation suggested that critical sensitivity might be a possible common feature prior to catastrophe in heterogeneous brittle media (XIA et al.2002). Critical sensitivity means that a system may become sensitive significantly as approaching its catastrophe transition point. The underlying mechanism behind critical sensitivity is the coupling effect between disordered heterogeneity on multiple scales and dynamical nonlinearity due to damage-induced stress redistribution (STEIN, 1999; XIA et al., 2000, 2002; WEI, et al. 2000). Critical sensitivity may provide a clue to the prediction of catastrophe transition, such as material failure and great earthquakes, provided the sensitivity of the system is measurable or can be monitored.

Rupture in disordered heterogeneous brittle media can be considered as catastrophe transition in driven disordered, nonlinear threshold systems. In this paper we present an analytical approach to reveal the mechanism of critical sensitivity, based on mean field approximation and damage relaxation time model, and compare the results with experimental data on rock rupture. It is found that the critical sensitivity is a common feature of catastrophe in driven nonlinear threshold systems, which seems to be supported by the rock rupture experiments. Hence, the critical sensitivity may provide a clue to catastrophe prediction.

\section{Physical Model of Heterogeneous Brittle Media}

We consider a macroscopic system comprised of numerous interacting, nonlinear mesoscopic units. The scale of the mesoscopic units corresponds to the intrinsic characteristic scale of heterogeneous structure, and the span between macroscopic and mesoscopic scales is about $10^{3}-10^{6}$ in typical cases of rupture. The mesoscopic units are identical statistically. The mesoscopic heterogeneity of material property is attributed to the quenched disorder of broken threshold of units, i.e., a predefined threshold $\sigma_{\mathrm{c}}$ is assigned to each unit. $\sigma_{\mathrm{c}}$ follows a statistical distribution function $h\left(\sigma_{\mathrm{c}}\right)$, which is normalized as

$$
\int_{0}^{\infty} h\left(\sigma_{c}\right) d \sigma_{c}=1
$$

The system is subjected to nominal driving force $\sigma_{0}$, which is adopted as macroscopic, external parameter. A unit breaks as the driving force $\sigma$ on it becomes higher than its threshold. When a unit breaks, it will be excluded from the 
distribution function. Therefore, we introduce a time-dependent distribution function of intact elements $f\left(\sigma_{\mathrm{c}}, t\right)$ with initial condition

$$
f\left(\sigma_{c}, t\right)=h\left(\sigma_{c}\right) .
$$

In such a system, stress redistribution induced by damage evolution plays an essential role in nonlinear dynamics. In most cases, the stress redistribution leads to stress fluctuations in stress pattern, and the coupling effect between inhomogeneous stress and the heterogeneity of the threshold of units leads to considerably more complex behavior of catastrophe. In order to take an analytical approach, we adopt a simple model based on a globally mean-field approximation. According to the mean-field approximation, the nominal driving force $\sigma_{0}$ is loaded uniformly on all intact units, i.e., the real driving force is determined by

$$
\sigma=\frac{\sigma_{0}}{1-p}
$$

This is a widely used formula in damage mechanics, where $p$ is damage fraction calculated from

$$
p=1-\int_{0}^{\infty} f\left(\sigma_{c}, t\right) d \sigma_{c}
$$

The evolution of distribution function $f\left(\sigma_{c}, t\right)$ is suggested to follow an equation based on relaxation time model:

$$
\frac{\partial f\left(\sigma_{c}, t\right)}{\partial t}=-\frac{f\left(\sigma_{c}, t\right)}{\tau}
$$

where $\tau$ is characteristic time of damage relaxation. Generally speaking, $\tau$ decreases with increasing $\frac{\sigma}{\sigma_{c}}$. For simplicity, we assume

$$
\tau= \begin{cases}\infty, & \text { as } \sigma<\sigma_{c} \\ \tau_{D}\left(\frac{\sigma}{\sigma_{c}}\right)^{-q}, & \text { as } \sigma_{M} \geq \sigma \geq \sigma_{c}, \\ 0, & \text { as } \sigma>\sigma_{M}\end{cases}
$$

Such a model implies that the damage relaxation can be characterized by three time scales: for $\sigma<\sigma_{c}$ the damage appears as a very slow relaxation process, and we simply assume $\tau \rightarrow \infty$; for very high driving force, the damage becomes a very fast relaxation process, and the relaxation time is nearly zero; this corresponds to the catastrophic rupture, in addition, it also means a cutoff of distribution function $h\left(\sigma_{c}\right)$ at $\sigma_{c}=\sigma_{M}$; and for the intermediate case, the damage relaxation can be described by a finite relaxation time which depends on $\frac{\sigma}{\sigma_{c}}$ and $q$ is a positive parameter. The rupture in real heterogeneous brittle media usually presents catastrophe transition, i.e., the transition of evolution mode from globally stable accumulation of damage to catastrophic rupture. Thus, macroscopically the evolution is characterized by two 
time scales: a long time scale for damage accumulation and a very short time scale for catastrophe. Such a behavior can be modeled by the above-mentioned damage relaxation model with three characteristic time scales. The concern in this paper is with the common behaviors prior to $\sigma=\sigma_{M}$ rather than the value of $\sigma_{M}$.

In order to reveal the main mechanism of critical sensitivity, we adopt a simplified and ideal model which only takes the coupling between dynamical nonlinearity and mesoscopic heterogeneity into account, and a series of complicated effects are neglected. Subsequently, the model cannot describe a real system quantitatively.

\section{Critical Sensitivity in the Case of Quasistatic Loading}

For the case of quasistatic and monotonic loading; the damage evolution will reach equilibrium at each nominal driving force. $\sigma_{0}$ We denote the solution of equation (5) by $F\left(\sigma_{c}, \sigma_{0}\right)$ which is expressed by (see Fig. 1)

$$
F\left(\sigma_{c}, \sigma_{0}\right)= \begin{cases}0, & \text { for } \sigma_{c}<\frac{\sigma_{0}}{1-P} \\ h\left(\sigma_{c}\right), & \text { for } \sigma_{c} \geq \frac{\sigma_{0}}{1-P}\end{cases}
$$

where $P=P\left(\sigma_{0}\right)$ is equilibrium damage fraction at $\sigma_{0}$ which is the solution of the following equation, which is derived from the definition (4) in the case of quasistatic and monotonic loading:

$$
P\left(\sigma_{0}\right)=\int_{0}^{\frac{\sigma_{0}}{1-P\left(\sigma_{0}\right)}} h\left(\sigma_{0}\right) d \sigma_{c}
$$

The response of the system to increasing driving force can be defined in terms of various variables, for instance, damage fraction or cumulative energy release. In this paper, it is defined by

$$
R\left(\sigma_{0}\right)=\frac{d E\left(\sigma_{0}\right)}{d \sigma_{0}}
$$

where $E\left(\sigma_{0}\right)$ is the cumulative energy release.

\section{Figure 1}

Critical sensitivity in driven nonlinear threshold systems in the case of quasistatic loading based on meanfield approximation and damage relaxation model. $h\left(\sigma_{c}\right)$ is Weibull distribution function with modulus $m=2$ and 5, respectively. (a) The equilibrium distribution function $F\left(\sigma_{c}, \sigma_{0}\right)$ at $\sigma_{0}=\sigma_{o f}-0, m=2$. (b) The equilibrium distribution function $F\left(\sigma_{c}, \sigma_{0}\right)$ at $\sigma_{0}=\sigma_{o f}-0, m=5$. (c) The cumulative energy release (the lower curves) and the sensitivity (the upper curves). $\sigma_{0}$ is the nominal driving force and the cumulative energy release $E$ is normalized by $E_{\text {cum }}=\int_{0}^{\sigma_{o f}-0} R\left(\sigma_{0}\right) d \sigma_{0}$. The cumulative energy release (the lower curves) displays a catastrophe at $\sigma_{0}=\sigma_{o f}$. The sensitivity $S$ (the upper curves) is defined by equation (12) and normalized by $S\left(\sigma_{0}=0\right)=m+1 . S$ increases significantly prior to catastrophe, suggesting critical sensitivity. 
(a)

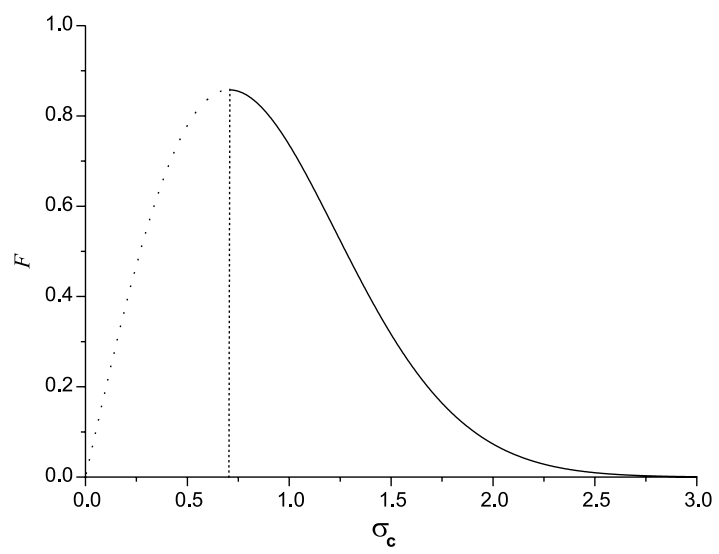

(b)

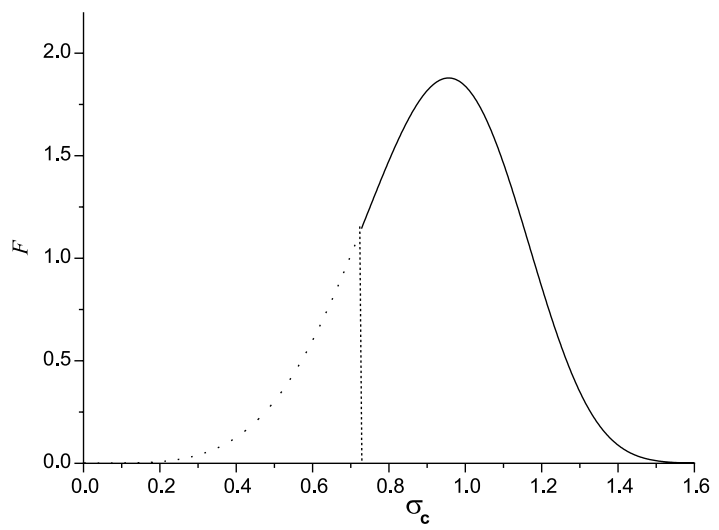

(c)

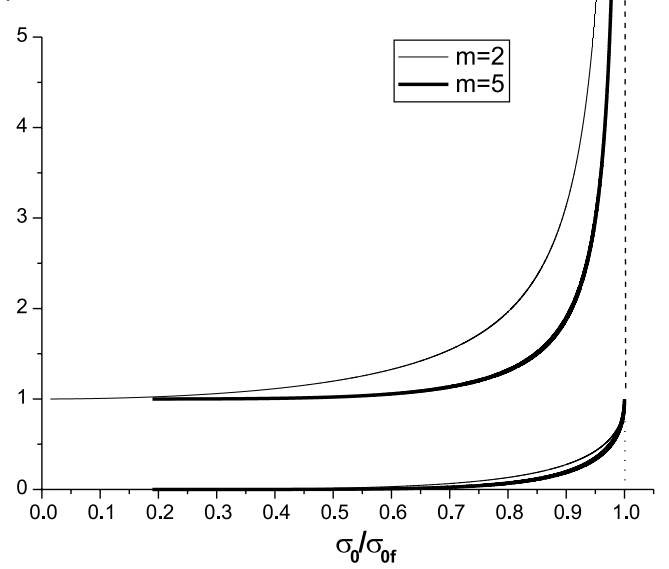


The catastrophe transition can be determined by the condition

$$
\frac{d P\left(\sigma_{0}\right)}{d \sigma_{0}}=\infty
$$

The response $R$ displays catastrophe transition at $\sigma_{0}=\sigma_{o f}$ We will denote the damage fraction at catastrophe transition point by $P_{c}$

For $\sigma_{0}<\sigma_{o f}$ the equilibrium state of the system evolves continuously with increasing $\sigma_{0}$ and $P\left(\sigma_{0}\right)<P_{c}$ This is the stage of stable accumulation of damage. However, at $\sigma_{0}=\sigma_{o f}$ the equilibrium state jumps to global failure state $(\mathrm{P}=1)$ displaying catastrophe transition, i.e., the system falls into a situation of selfsustained catastrophic failure.

In order to measure the sensitivity of the response to nominal driving force, we define the sensitivity as

$$
S\left(\sigma_{0}\right)=\frac{\sigma_{0}}{R\left(\sigma_{0}\right)} \frac{d R\left(\sigma_{0}\right)}{d \sigma_{0}} .
$$

A highly sensitive state is characterized by $S \gg 1$. Since there may be various definitions of the response, there also will be various definitions of the sensitivity. In particular, heterogeneous brittle media present sample specificity; the sensitivity cannot be represented by macroscopically average properties, e.g., free energy, however the definition (11) of sensitivity is available for real systems.

When the initial distribution function is assumed to be a Weibull distribution function

$$
h\left(\sigma_{c}\right)=m \sigma_{c}^{m-1} \exp \left(-\sigma_{c}^{m}\right) .
$$

the cumulative energy release $E\left(\sigma_{0}\right)$ and sensitivity $S\left(\sigma_{0}\right)$ for the global mean field model are presented in Figure 1 (for a Weibull distribution function with modulus $m=2$ and $m=5$ ). Weibull distribution is a widely used function to characterize the diversity of material properties. The cumulative energy release shows that the evolution displays a catastrophe transition at $\sigma_{0}=\sigma_{o f}=(m e)^{\frac{1}{m}}$ and $P_{c}=1-e^{-1 / m}\left(\sigma_{o f}=0.4289\right.$ and $P_{c}=0.3935$ for $m=2$, and $\sigma_{o f}=0.5934, P_{c}=0.1813$ for $\left.m=5\right)$. In the case of $\sigma_{0} \sim 0$, we derive $\mathrm{S} \sim \mathrm{m}+1$, corresponding to an insensitive state. However, we can see that $S \rightarrow \infty$ as $\sigma_{0} \rightarrow \sigma_{\text {of }}$ which implies that the system becomes sensitive significantly as it approaches its catastrophe transition point. Such a feature is called critical sensitivity, which is an important precursor of catastrophe.

\section{The Effects of Loading Rate on Catastrophe and Critical Sensitivity}

The assumption of quasistatic loading is valid only for the case that the characteristic time of loading is much longer than that of damage relaxation. Otherwise, the effects of time-dependent damage relaxation should be taken into account. 
For the case with time-dependent nominal driving force $\sigma_{0}(t)$ the distribution function $f\left(\sigma_{c}, t\right)$ can be solved from equations (5) and (6). The energy release rate is given by

$$
R(t)=\frac{N}{2 k} \frac{\sigma_{0}^{2}(t)}{(1-p(t))^{2}} \frac{d p(t)}{d t},
$$

where $N$ is the total of mesoscopic units and $\mathrm{k}$ is the stiffness of units, which is assumed to be identical for all units. We will consider the continuous limitation that $\lim _{N \rightarrow \infty} \frac{N}{k}=$ const. The sensitivity of response of the system to nominal driving force can be measured by

$$
S(t)=\frac{\sigma_{0}(t)}{R(t)} \frac{\frac{d R(t)}{d(t)}}{d t(t)}
$$

Now we consider the case that the nominal stress increases with time linearly:

$$
\sigma_{0}(t)=\frac{\alpha \sigma_{o f}}{\tau_{D}} t,
$$

where $\sigma_{o f}$ is the catastrophe transition point in the case of quasi static loading, $\alpha$ is a constant. The quasi-static loading corresponds to the limitation $\alpha \rightarrow 0$.

Figure 2 illustrated the cumulative energy release and the sensitivity for the models with $\sigma_{M}=4$ and $q=0,1$ and 2, respectively. The loading rate is characterized by $\alpha=0.1$ and the initial threshold distribution function is adopted as Weibull distribution function with modulus $m=3$. The cumulative energy release presents a continuous increase followed by a finite jump $\Delta E$. This corresponds to a transition from continuous accumulation of damage to catastrophic rupture. The jump of cumulative energy release is expressed by

$$
\Delta E=\frac{N}{2 k} \sigma_{M}^{2}\left(1-D_{M}\right),
$$

where $D_{M}$ is damage fraction at $\sigma=\sigma_{M}$. From Figure 2 we can see that prior to catastrophic rupture, sensitivity increases significantly. Therefore, the critical sensitivity is also a precursory feature of the catastrophic rupture when the loading rate is considered. In Figure 2 the downward arrow indicates the catastrophe transition point in the case of quasistatic loading. It is found that both the catastrophic rupture and the critical sensitivity are delayed by the loading rate effect, comparable with the case of quasistatic loading. This is because the criticality of the system is determined by the coupling between the external condition and the state of the system. The appearance of catastrophe and critical sensitivity is related to the evolution of the system.

It is noted that there are differences in curves of sensitivity for the models with different $q$ values. The higher the $q$ value is, the faster the relaxation process becomes. 
(a)

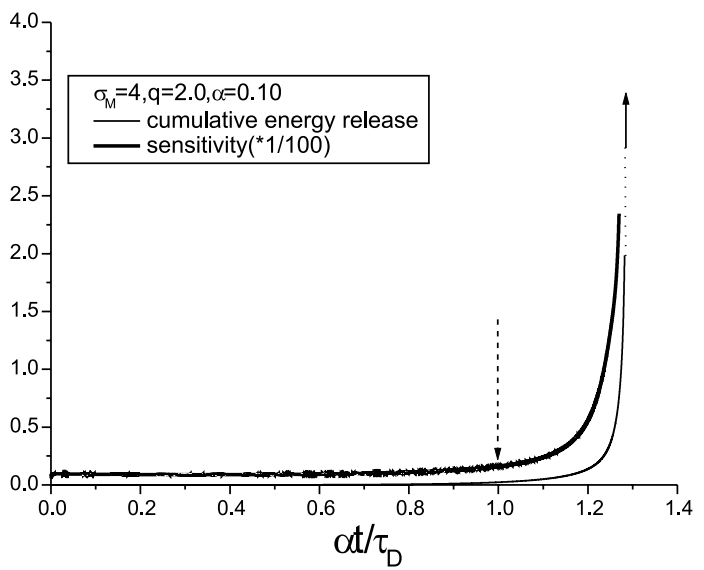

(b)

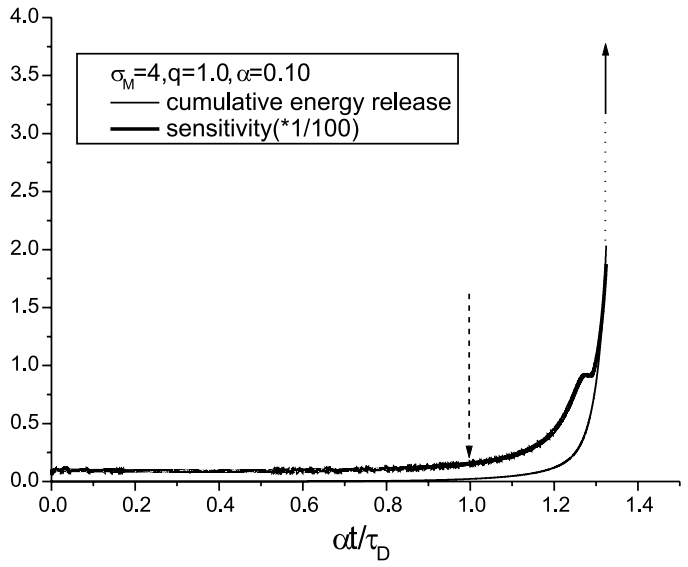

(c)

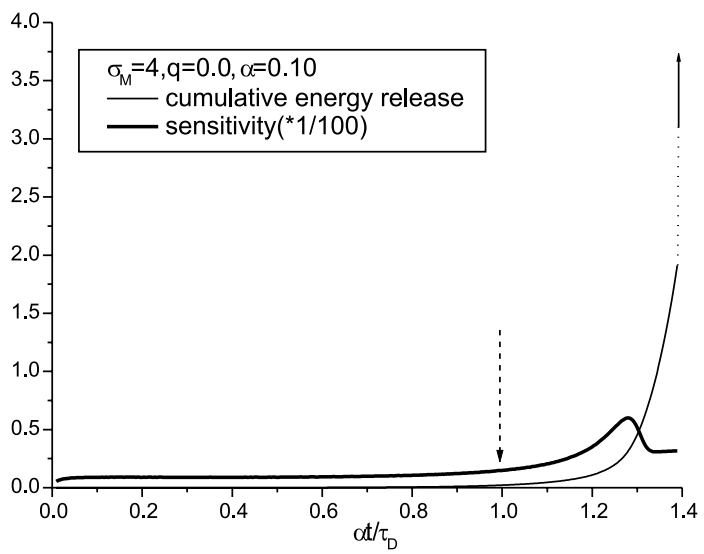


The quasistatic loading corresponds to the limitation $q \rightarrow \infty$. The characteristics of the sensitivity curve for $q=2$, the case of faster relaxation, are similar to that for quasistatic loading, i.e., it shows monotonic increase. However, the sensitivity curve for $q=0$, the case of slower relaxation, presents different characteristics, i.e., it shows a peak prior to catastrophic rupture. The characteristics of the sensitivity curve are determined by the coupling and competition between the effects of loading, damage relaxation and dynamical nonlinearity (redistribution of driving force). From equations (13)-(15), the sensitivity $S$ can be expressed as

$$
S=2+\left(\frac{2}{1-p} \frac{d p}{d t}+\left(\frac{d p}{d t}\right)^{-1} \frac{d^{2} p}{d t^{2}}\right) t .
$$

The factor $t$ in the right hand of the equation derives from the linear loading, the time-dependent behavior of the damage $p(t)$ reflects the evolution of distribution function $f\left(\sigma_{c}, t\right)$ Figure 3 depicts the evolution of $f\left(\sigma_{c}, t\right)$ prior to the catastrophic rupture for various damage relaxation models, i.e., for the models with $q=0$ and, $q=2$ respectively. We can see that the damage relaxation process for the model with $q=2$ is faster than that for the model wit $q=0$.

\section{Effects of Stress Fluctuations on Catastrophe and Critical Sensitivity}

In mean field approximation, stress fluctuations are neglected. However, the stress fluctuations play an essential role in catastrophe (WEI, et al 2000). As we take the stress fluctuations into account, the problem becomes far more complicated. Numerical results revealed that the evolution also presents catastrophe transition although the catastrophe displays sample-specificity. Regardless, it is also found that the critical sensitivity is in reality the common precursory feature of catastrophe in the case with stress fluctuations, although the sensitivity manifests strong fluctuations, see reference XIA et al (2002).

\section{Critical Sensitivity in Rock Rupture Experiments}

Now we give results of rock rupture experiments YIN et al (2002) on samples of $1050 * 400 * 100 \mathrm{~mm}^{3}$ (for marble and gneiss) and $1050 * 400 * 150 \mathrm{~mm}^{3}$ (for sand-

Figure 2

Cumulative energy release and sensitivity in the case of time-dependent nominal driving force $\sigma_{0}(t)=\alpha \frac{\sigma o f}{\tau_{0}} t$ with $\alpha=0.1 . h\left(\sigma_{c}\right)$ is Weibull distribution function with modulus $m=3$. (a) $\sigma_{M}=4, q=2$; (b) $\sigma_{M}=4$, $q=1$; (c) $\sigma_{M}=4, q=0$. The upward arrows indicate the catastrophic rupture and the downward arrows indicate the catastrophe transition point in the case of quasistatic loading. 

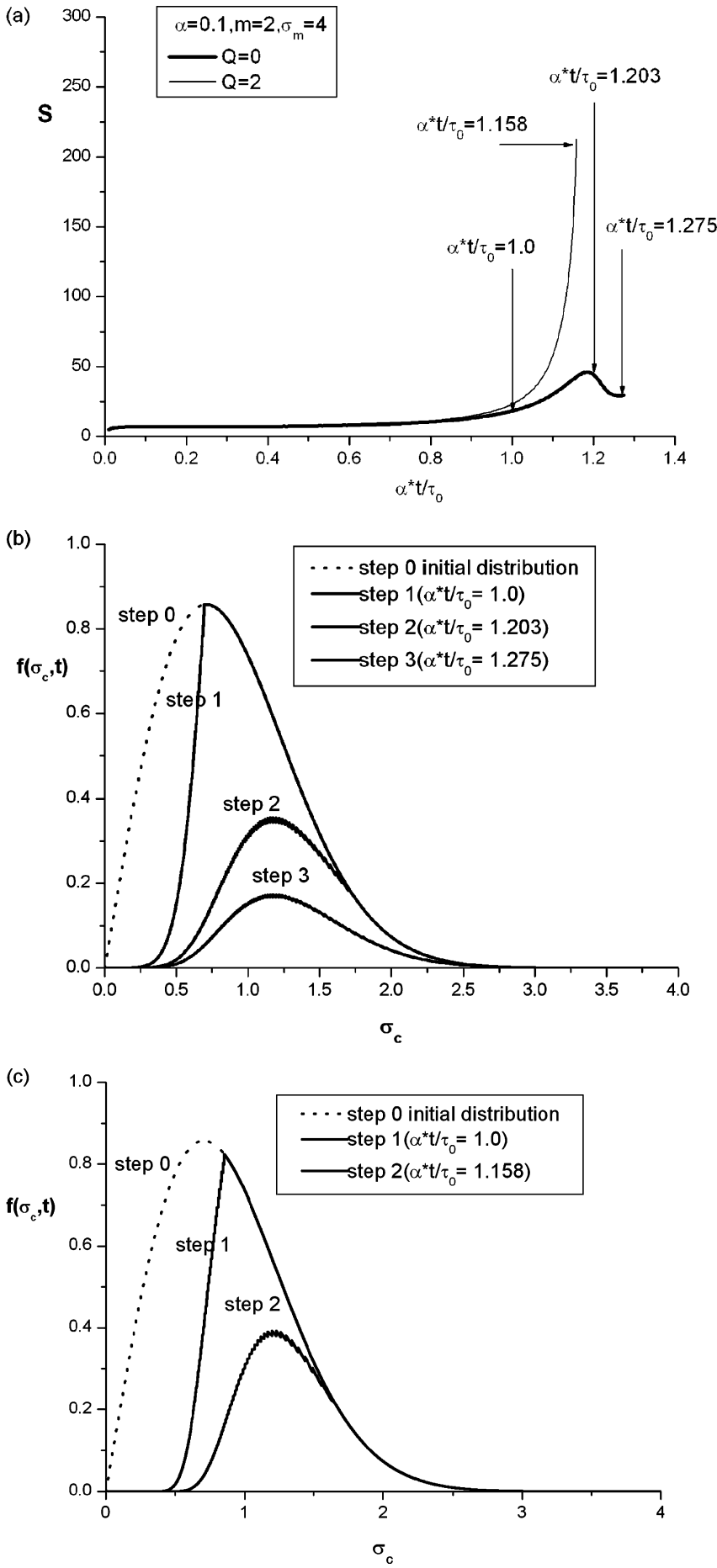
stone). The samples were compressed until rupture. The behavior shows catastrophe transition from the accumulation of mesoscopic damage to macroscopic rupture. Mesoscopic damage is characterized by energy release recorded by acoustic emission (AE). Typically, the total of recorded AE events is about $4 * 10^{5}$ in the experiments. The sensitivity of energy release to external load can be defined by

$$
S=\frac{F}{R} \frac{\Delta R}{\Delta F}
$$

where $F$ is the external load, $R$ is the energy release rate, and $\Delta R$ is the increment of energy release rate corresponding to the increment of external load $\Delta F$. The experimental results are shown in Figure 4. It is note worthy to compare the experimental results, Figure 4, with the theoretical results, Figure's 1-3. Theoretical results of sensitivity are expressed by a deterministic and smooth curve. This is related to the continuous limitation, mean-field approximation and relaxation time model. The experimental and numerical results of sensitivity display fluctuations (strong fluctuations imply high sensitivity) and sample specificity (the time series of sensitivity are different from each other for samples identical macroscopically). Such behavior is related to the fact that the mesoscopic damage events are discrete and stochastic in a real system or numerical models with stress fluctuations. Nonetheless, the rock rupture experiments seem to support the critical sensitivity: the sensitivity increases significantly prior to the catastrophic rupture point.

\section{Summary}

Rupture in heterogeneous brittle media is a complicated phenomenon, and rupture prediction, e.g. earthquake forecast, is a difficult problem of vital societal concern. Critical sensitivity as a universality of catastrophe transition in driven nonlinear threshold systems may help us to capture the essence of catastrophe transition. In some cases, the sensitivity might be measurable. Monitoring the sensitivity of the system may yield helpful clues to rupture prediction.

\section{Figure 3}

The evolution of distribution function $f\left(\sigma_{c}, t\right)$ prior to the catastrophic rupture. $h\left(\sigma_{c}\right)$ is Weibull distribution function with modulus $m=2$. The system is subjected to nominal driving force $\sigma_{0}(t)=\alpha \frac{\sigma_{o f}}{\tau_{0}} t$ with $\alpha=0.1$ (a) The sensitivity $S$ for relaxation model with $\sigma_{M}=4$ and $q=0$ and 2, respectively; (b) The evolution of distribution function $f\left(\sigma_{c}, t\right)$ for damage relaxation model with $q=0$; (c) The evolution of distribution function $f\left(\sigma_{c}, t\right)$ for damage relaxation model with $q=2$. 
(a)

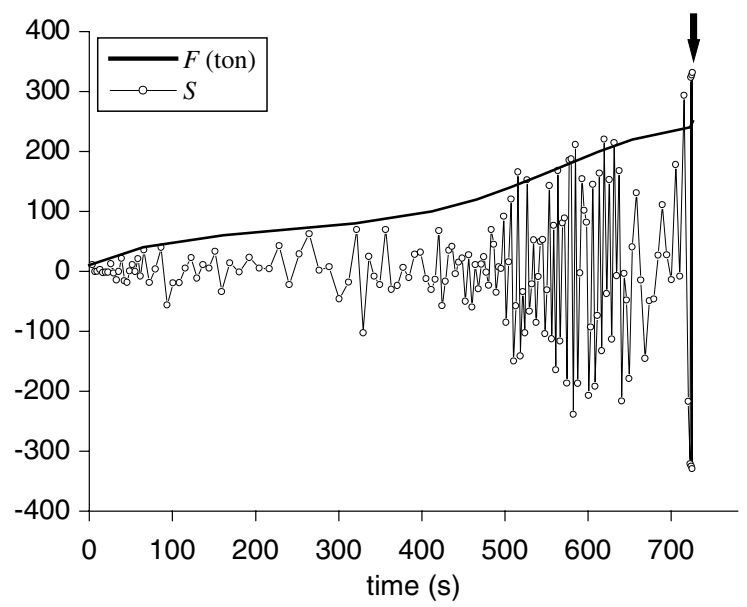

(b)

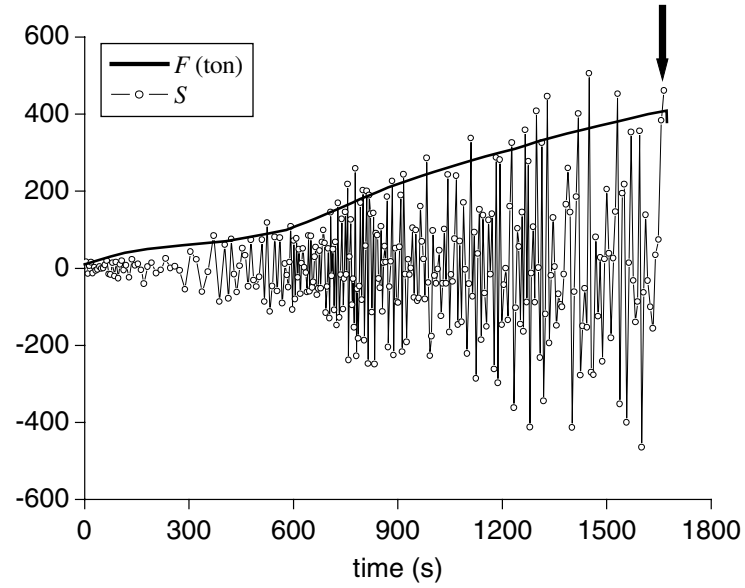

(c)

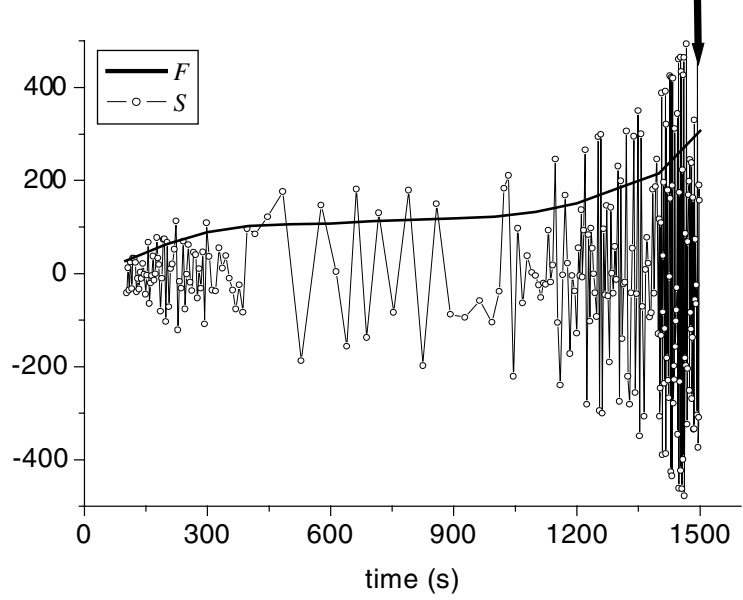


Figure 4

Critical sensitivity in a rock rupture experiment. The curves are axial force $F(t)$ and the sensitivity $S(t)$ respectively, and $S(t)$ is calculated from the data of acoustic emission. The total of recorded AE events is about $4 * 10^{5}$. The arrow indicates the catastrophic rupture point. The experimental results seem to support the critical sensitivity: the sensitivity increases significantly prior to the catastrophic rupture point. (a) Gneiss; (b) Sandstone; (c) Marble.

\section{Acknowledgement}

This work is supported by the Special Funds for Major State Research Project (G2000077305) and the National Natural Science Foundation of China (No. 10372012, 10047006, 10232040, 10232050).

\section{REFERENCES}

BaI, Y. L., Lu, C. S., Ke, F. J., and XIA, M. F. (1994), Evolution Induced Catastrophe, Phys. Lett. A. 185, 196-200.

Ben-Zion, Y. and Sammis, C. G. (2003), Characterization of Fault Zones, Pure Appl. Geophys. 160, 677-715.

Curran, D. R., Seaman, L., and Shockey, D. A. (1997), Dynamic Failure of Solids, Phys. Rep. 147, 253388.

Curtin, W. A. (1997), Toughening in Disordered Brittle Materials, Phys. Rev. B 55, 11270-11276.

Garcimartin, A. Guarino, A. Bellon, L. and Ciliberto, S. (1997), Statistical Properties of Fracture Precursors, Phys. Rev. Lett. 79, 3202-3205.

Jaume, S. C. and Sykes, L. R. (1999), Evolving Towards a Critical Point: A Review of Accelerating Seismic Moment/Energy Release Prior to Large and Great Earthquakes, Pure Appl. Geophys. 155, 279-305.

Meakin, P. (1991), Models for Material Failure and Deformation, Science 252, 226-234.

Rundle, J. B., Klein, W., Tiampo, K. F., and Gross, S. (2000), Linear Pattern Dynamics in Nonlinear Threshold Systems, Phys. Rev. E 61, 2418-2432.

Rundle, J. B., Klein, W., Tiampo, K. F., and Gross, S. (2000), Dynamics of Seismicity Patterns in Systems of Earthquake Faults, Geocomplexity and the Physics of Earthquakes, AGU Monograph, Washington, D.C.,pp 127-146.

Sahimi, M. and Aвавi, S. (1993), Mechanics of Disordered Solid. III. Fracture Properties, Phys. Rev. B 47, $713-722$.

Stein, R. S. (1999), The Role of Stress Transfer in Earthquake Occurrence, Nature 402, 605-609.

Tiampo, K. F., Rundle, J. B., Klein, W., Mcginnis, S., and Gross, S. J. (2000), Observation of Systematic Variations in Non-local Seismicity Patterns from Southern California, Geocomplexity and the Physics of Earthquakes, AGU Monograph, Washington, D. C.

WeI, Y. J., XIA, M. F., Ke, F. J., YIN, X. C. and BAI, Y. L. (2000), Evolution Induced Catastrophe and its Predictability, Pure Appl. Geophys. 157,1945-1957.

Wyss, M., Aceves, R. L., Park, S. K., Geller, R .J., Jackson, D. D., Kagan, Y. Y., and Mulargia, F. (1997), Cannot Earthquakes Be Predicted? Science 275, 480-490.

XIA, M. F., KE, F. J., and BAI, Y. L. (1997), Threshold Diversity and Trans-scale Sensitivity in a Nonlinear Evolution Model, Phys. Lett. A 236, 60-64.

XIA, M. F., Ke, F. J., and BAI, Y. L. (2000), Evolution Induced Catastrophe in a Nonlinear Dynamical Model of Materials Failure, Nonlinear Dynamics 22,205-224.

XIA, M. F., SonG, Z. Q., Xu, J. B., ZhaO, K. H., and BaI, Y. L. (1996), Sample-specific Behavior in Failure Models of Disordered Media, Communication in Theoretical Physics. 25, 49-54.

XIA, M. F., WeI, Y. J., KE, F. J., and BAI, Y. L. (2002), Critical Sensitivity and Trans-scale Fluctuations in Catastrophic Rupture, Pure Appl. Geophys. 159, 2491-2509. 
Yin, X. C., Yu, H. Z., Kukshenko, V., Xu, Z. Y., Li, Q., Xia, M. F., Li, M., Pen, K. Y., and Elizarov, S., Load-Unload Response Ratio (LURR) and Accelerating Moment/Energy Release (AM/ER) and State Vector, 3rd ACES International Workshop, May 5-10, 2002, Island of Maui, State of Hawaii, USA.

(Received September 27, 2002, revised January 27, 2003, accepted February 10, 2003)

(1) To access this journal online:

(20) http://www.birkhauser.ch 